\title{
Atribuições do farmacêutico em unidade de assistência de alta complexidade em oncologia
}

\author{
Pharmacist's role in service of high complexity in oncology
}

Hozana SANTOS ${ }^{1 *}$, Giumara BATISTA², Gabriel MOTA, Max MARTINS ${ }^{4}$, Sheila NUNES ${ }^{5}$.

${ }^{I}$ Faculdade Pitágoras (Unidade São Luis), São Luís - MA, Brasil. ${ }^{2}$ Faculdade de Imperatriz (FACIMP), Imperatriz - MA, Brasil . ${ }^{3}$ Faculdade de Imperatriz (FACIMP), Imperatriz -MA, Brasil . ${ }^{4}$ Universidad San Francisco Xavier de Chuquisaca, Sucre-Bolivia ${ }^{5}$ Universidade Estadual do Maranhão, Imperatriz - MA, Brasil. *E-mail: hozana_9@hotmail.com

\begin{abstract}
In recent years, the pharmaceutical skilled in oncology has proved increasingly important and ready to perform their role in society. He is the main instrument of Pharmacotherapy and important in various stages of anticancer therapy. With the goal to identify the responsibilities, powers and operation of the pharmacist in oncology service recently implemented in the second largest city in the state of Maranhão proposed to conduct this study. Data were collected through interviews and visits with the pharmacist responsible for pharmacy oncology hospital. The distribution of the data collected was consolidated in terms of installed capacity, occupation and activities. Activities observed in the performance of private professional handling of cytostatic agents. He emphasized the use of Doxorrubirina, Daunoblostina, Paclitaxel, Citorabina, Daumoclastma, Cytarabine, Anastrozole, Uimorelbina, Busulfan, oxaliplatin among others. It was possible to follow the routine oncology pharmacy and highlight the importance of professional evaluation from the prescription, dispensing, handling, evaluation and registration of RAM among other activities.
\end{abstract}

KEYWORDS: Pharmacy; Medical Oncology; Professional; Practice clinical.

\section{RESUMO}

Nos últimos anos, o farmacêutico especializado em oncologia, tem-se mostrado cada vez mais importante e preparado para exercer seu papel diante da sociedade. Ele é o principal instrumento da Farmacoterapia e importante em várias etapas da terapia antineoplásica. Com objetivo de identificar as atribuições, competências e atuação do farmacêutico no serviço de oncologia recém-implantado na segunda maior cidade do estado do Maranhão se propuseram a realização desta pesquisa. Os dados foram coletados através de visitas e entrevista com o farmacêutico responsável pela farmácia de oncologia do hospital. A distribuição dos dados levantados foi consolidada em termos de capacidade instalada, categoria profissional e atividades desenvolvidas. Nas atividades desenvolvidas observou-se a atuação privativa do profissional na manipulação dos agentes citostáticos. Destacou-se a utilização de citotásticos como Doxorrubirina, Daunoblostina, Paclitaxel, Citorabina, Daumoclastma, Citarabina, Anastrozol, Uimorelbina, Bussulfano, Oxaliplatina dentre outros. Foi possível acompanhar a rotina da farmácia oncológica e destacar a importância do profissional desde a avaliação da prescrição médica, dispensação, manipulação, avaliação e registro de RAM entre outras atividades.

PALAVRAS CHAVE: Farmácia; Oncologia; Profissional; Prática clínica 


\section{LISTA DE ABREVIAÇÕES:}

\section{AF Atenção Farmacêutica}

ANVISA Agência Nacional de Vigilância Sanitária

CFF Conselho Federal de Farmácia

CONASS Conselho Nacional de Secretários de Saúde

DOU Diário Nacional da União

EMTA Equipe Multidisciplinar de Terapia Antineoplásica

EPC Equipamentos de Proteção Coletiva

EPI Equipamentos de Proteção Individual

FTN Formulário Terapêutico Nacional

HSR Hospital São Rafael

OPAS Organização Pan-Americana de Saúde

PDCT Protocolos Clínicos e as Diretrizes Terapêuticas

RAM Reações Adversas Medicamentosas

RDC Resolução de Diretoria Colegiada

RENAME Relação Nacional de Medicamentos Essenciais

SOBRAFO Sociedade Brasileira de Farmacêuticos em Oncologia

SUS Sistema Único de Saúde

UNACOM Unidade de Assistência de Alta Complexidade em Oncologia

\section{INTRODUÇÃO}

Nos últimos anos, o farmacêutico com especialização em oncologia tem-se mostrado cada vez mais importante e preparado para atuar na farmacoterapia em várias etapas da terapia antineoplásicas e de outros procedimentos farmacológicos. Esse cenário crescente se deve a Resolução 288/96 editada pelo Conselho Federal de Farmácia (CFF) que implica na manipulação de drogas antineoplásicas, exclusivamente pelo farmacêutico (1), acarretando no aumento significativo da atuação desse profissional na área oncológica e consequentemente recuperando-o das mãos dos enfermeiros.

$\mathrm{Na}$ área oncológica a Portaria 3.535/98, do Ministério da Saúde que estabelece critérios para cadastramento de centros de atendimento em oncologia e determina que todo serviço de alta complexidade no tratamento do câncer, cadastrado no Sistema Único de Saúde (SUS), deve contar com um farmacêutico, no caso de manipulação de quimioterápicos (2). Assim justifica-se a grande importância de tal profissional nas atividades desenvolvidas dentro da farmácia hospitalar no setor de oncologia. Portanto, tornou-se essencial a procura dos mesmos por conhecimento técnico - científico visando uma qualificação mais específica na tão complexa e vasta área oncológica.

A RDC 220/2004 prevê a inserção do farmacêutico oncológico na Equipe Multidisciplinar e Interdisciplinar do serviço oncológico, tornando-o assim, uma peça fundamental com atribuições desde a aquisição dos medicamentos até o seu devido descarte (3). Proporcionando ao profissional farmacêutico uma presença importante nos serviços de quimioterapia em todo o Brasil (4).

Além disto, o farmacêutico precisa estar constantemente atento às reações e toxicidade dos medicamentos citostáticos. Afinal, a toxicidade ocorre em média em $6 \%$ a $10 \%$ dos pacientes hospitalizados (5). Norteando desta maneira, a necessidade não é apenas da assistência farmacêutica, mas também de forma abrangente a atenção farmacêutica (AF). Pois por meio da AF, é possível obter desempenhos significativos quanto à terapêutica medicamentosa, da autoconfiança dos pacientes e preservação da autonomia dos mesmos (4).

O farmacêutico da área de oncologia, além de participar da gestão da farmácia também discute sobre procedimentos oncológicos mais rebuscados, com a equipe multidisciplinar (6). O número e a localização de tais profissionais são bastante inexpressivos, sendo a procura muito maior que a oferta. Atribui-se a esse cenário à falta de qualificação e por não haver capacitação acadêmica especifica.

Tangente a isso, em 2001 foi criado a Sociedade Brasileira de Farmacêuticos em Oncologia (SOBRAFO), fortalecendo desta forma a classe e dando-lhes um apoio técnico-científico (7). Na área farmacêutica o delineamento rumo à oncologia apenas deu-se no ano de 1990 e a SOBRAFO tornou-se uma sociedade formal de referência em oncologia a partir de 2001(8), dados estes, relevantes quando se reflete no período que antecede tais anos.

Atualmente é de grande relevância conhecer a real atuação deste profissional em especial nos centros de referencia do país. Com objetivo de identificar as atribuições, competências e atuação do farmacêutico no serviço de oncologia recém implantado na segunda maior cidade do estado do Maranhão se propuseram a realização desta pesquisa. 


\section{MATERIAIS E MÉTODOS}

A pesquisa foi realizada na cidade de Imperatriz no Estado do Maranhão. O município ocupa hoje a posição de segundo maior centro populacional, comercial, econômico, político e cultural do estado e, absorve a missão de distribuidora de bens e serviços para uma ampla área que compreende o sul do Pará, oeste do Maranhão e norte do Tocantins, considerada na área da saúde referência na região.

Em 2011 foi implantado o serviço de oncologia no Hospital São Rafael (HSR) credenciado como Unidade de Assistência de Alta Complexidade em Oncologia (UNACOM), a segunda no estado a receber tal credenciamento (9) e a primeira fora da capital do estado. Oferecendo diagnóstico e tratamento quimioterápico e cirúrgico aos pacientes portadores de cânceres. Através de visitas e entrevista realizadas entre setembro a novembro de 2010, com o farmacêutico responsável pela farmácia do setor de oncologia do hospital e com auxilio de questionário foi possível reproduzir de modo fiel às tarefas executadas pelo profissional.

As informações coletadas foram descritas, comparadas e confrontadas com a realidade de outros centros para que fosse possível identificar de forma clara as atribuições de um farmacêutico dentro da farmácia especializada em oncologia, sob a perspectiva do profissional e da realidade existente dentro do hospital.

\section{RESULTADOS E DISCUSSÃO}

A distribuição dos dados levantados foi consolidada em termos de capacidade instalada, categoria profissional e atividades desenvolvidas. O serviço de farmácia do Hospital tem sob sua responsabilidade atividades administrativas e clínico-assistenciais, visando o uso racional de medicamentos para a redução de custos e eventos adversos.

Quanto à capacidade instalada a farmácia comporta as áreas destinada à Central de Manipulação de Quimioterápicos (CMQ), armazenamento de medicações, área de termolábeis, atendimento dos prontuários. Áreas estas necessárias para funcionamento correto e indispensável para a farmacoterapia antineoplásica.

O corpo profissional da unidade é formado pelos componentes de equipe multidisciplinar, incluindo o farmacêutico. Nesta UNACOM, havia apenas um profissional farmacêutico que tinha todas as atribuições sob sua responsabilidade, sendo o mesmo auxiliado por outros profissionais.

Na UNACOM pesquisada, as atividades desenvolvidas envolvem desde seleção, aquisição dos medicamentos e avaliação da prescrição médica diária. A UNACOM conta com 21 leitos oncológicos, na rotina os medicamentos mais prescritos são os citotásticos como a Doxorrubirina, Daunoblostina, Paclitaxel, Citorabina, Daumoclastma, Citarabina, Anastrozol, Uimorelbina, Bussulfano, Oxaliplatina dentre outros.

Diariamente é avaliada pelo profissional farmacêutico a prescrição com a checagem do nome do paciente, número do prontuário, para evitar erros de preparo e de dispensação. E na avaliação destes protocolos prescritos, é verificado se a medicação está de acordo com o padronizado e realizado a checagem dos diluentes, verificando incompatibilidade físico-química com os citostáticos, verificando as doses e posologia prescrita.

A farmácia da UNACOM segue uma lista das classes terapêuticas descritas no Guia Digital de Medicamentos Genéricos (10) tais como o Anastrozol, Carboplatina, Cisplatina, Docetaxel, Doxorrubicina (Cloridrato), Finaste rida, Fluoruracila, Flutamida, Gencitabina (Cloridrato), Ifosfamida, Irinotecano (Cloridrato), Megestrol (Acetato), Metotrexato, Paclitaxel e Tamoxifeno (Citrato).

Quanto aos registros dos efeitos dos antineoplásicos, na unidade, foram observadas náuseas, vômitos, mal-estar, mucosites, plaquetopenia, sendo estas, as principais citadas pelo farmacêutico da unidade.

Estes sintomas são esperados em $100 \%$ dos casos, pois estão ligados aos mecanismos de ações das drogas (11). Há também, em alguns casos os efeitos inesperados, como as reações alérgicas, e os extravasamentos. De acordo com Martinez (12) o farmacêutico deve estar atento para que durante o tratamento não apareça o mínimo de problemas não desejados e, se aparecerem, resolvê-los com a ajuda do prescritor.

E como tais pacientes estão propícios as Reações Adversas Medicamentosas (RAM's), o farmacêutico, ao observar tais RAM's, toma várias medidas, como a notificação onde é possível aprimorar a identificação de reações adversas, possibilitando o acompanhamento ou busca em prontuários dos pacientes que utilizam tais medicamentos, incentivando a qualidade e a me- 
lhora do cuidado para garantir a segurança no uso de antineoplásicos (13).

$\mathrm{Na}$ a oncologia temos algumas definições para o que venha a ser, a atenção farmacêutica, dentre elas temos como sendo "a provisão responsável do tratamento farmacológico com o propósito de alcançar resultados terapêuticos concretos que melhorem a qualidade de vida do paciente", (13). Estes resultados são: Cura de uma enfermidade; eliminação ou redução de sintomas do paciente; interrupção ou retardamento do processo patológico; ou prevenção de uma enfermidade ou de um sintoma.

Quanto à relação dos medicamentos com uma terapia racional e de baixo custo, o fato encontrado foi que na UNACOM, não é levado em considerações tais processos, pois de acordo com o farmacêutico oncológico para os diferentes tipos de cânceres há seus respectivos protocolos de tratamento, cabendo aos médicos da EMTA (Equipe Multidisciplinar de Terapia Antineoplásica) escolher o mais adequado aos tratamentos.

Na unidade oncológica, o profissional farmacêutico, não encontra restrições pertinentes à sua atuação graças aos diversos protocolos da unidade. Contudo, a Agência Nacional de Vigilância Sanitária (ANVISA), após a emissão da RDC 222/04 (14), estabeleceu que o farmacêutico deve-se examinar a elaboração e adequação dos protocolos estabelecidos pela EMTA. Isso propicia meios de prevenção de erros e de melhoria dos tratamentos (15).

Na unidade oncológica as técnicas asséptica com medidas de segurança, utilizando-se de equipamentos de proteção individuais (EPI) e equipamentos de proteção coletivos (EPC) são comumente praticadas. Garantindo ótimas condições das soluções preparadas (16). Para Andrade (7), "o controle de qualidade deve ser continuo e diário, numa central de manipulação de quimioterápicos". Visando sempre as normas de segurança nacionais e internacionais.

No Brasil, o Formulário Terapêutico Nacional (FTN) da Relação Nacional de Medicamentos Essenciais (Rename) de 2006, publicado por meio de Portaria MS/GM n ${ }^{\circ} 1183$, de 09 de Setembro de 2008, contém informações científicas, isentas e embasadas em evidências sobre os medicamentos selecionados. São explicitadas as indicações terapêuticas, contra-indicações, precauções, efeitos adversos, interações, esquemas e cuidados de administração, orientação ao paciente, e aspectos farmacêuticos dos medicamen- tos selecionados (17). Outra extensão da Rename, no Brasil, são os Protocolos Clínicos e as Diretrizes Terapêuticas (PCDT). O protocolo terapêutico padroniza o emprego do medicamento, estabelecendo claras condições de uso para os profissionais de saúde (18). Tal protocolo é elaborado para medicamentos especialmente indicados pela importância epidemiológica da enfermidade que tratam ou pelo seu custo, ou pelo seu impacto sanitário, ou por questões de segurança de uso na população em geral ou em subgrupos.

No Brasil "a elaboração de Protocolos Clínicos tem sido realizada desde o início da década com a participação de muitos profissionais de distintas instituições gestoras (MS, Estados, Conselho Nacional de Secretários de Saúde (CONASS)); Organização Pan-Americana de Saúde (Opas); Hospitais, Instituições de Pesquisa e Universidades, Sociedades Médicas e usuários)" (14). E isto faz parte do processo de sua elaboração à participação de especialistas, a elaboração de uma primeira versão, submetida à Consulta Pública, cujas contribuições de especialistas nacionais e internacionais são avaliadas, sendo a versão final revisada e publicada no Diário Oficial da União (DOU).

Quanto à questão do gerenciamento de resíduos, os mesmos são acondicionados em sacos plásticos brancos leitosos pequenos e logo após as manipulações do dia são acondicionados em sacos plásticos brancos leitosos grandes, o que condiz e ao mesmo tempo confronta com a RDC 306/2004 (19), que em seu capitulo III, inciso 2, discorre sobre o acondicionamento de resíduos: "Consiste no ato de embalar os resíduos segregados, em sacos ou recipientes que evitem vazamentos e resistam às ações de punctura e ruptura. A capacidade dos recipientes de acondicionamento deve ser compatível com a geração diária de cada tipo de resíduo." Confronta no âmbito que diz que estes recipientes devem resistir a rupturas.

Logo após, de acordo com a legislação, os resíduos são transportados com carrinhos que também é requerido na RDC no seu capitulo III, artigo I inciso IV em que diz que: "Os recipientes para transporte interno devem ser constituídos de material rígido, lavável, impermeável, provido de tampa articulada ao próprio corpo do equipamento, cantos e bordas arredondados, e serem identificados com o símbolo correspondente ao risco do resíduo neles contidos e devem ser providos de rodas revestidas de material que reduza o ruído". 
Contudo, observa-se que o farmacêutico na área de oncologia possui, não apenas uma atribuição, mas sim, inúmeras que muitas vezes acarretam em sobrecarga de tarefas, já que em muitas instituições o número de profissionais é pequeno.

\section{CONCLUSÕES}

O farmacêutico é indispensável na unidade oncológica por ser o único habilitado a manipular os medicamentos quimioterápicos, onde deve sempre observar a portaria própria e as condições impostas pelos órgãos competentes. Mesmo diante da recente implantação do serviço, a UNACOM tem-se adequado as normas brasileiras e aos parâmetros indicados a acerca dos possíveis casos de toxicidade. Contudo, há necessidade de ampliação do quadro de profissionais.

\section{AGRADECIMENTOS}

Aos colaboradores do Hospital São Rafael, em especial ao farmacêutico responsável pela farmácia oncológica e ao diretor da unidade de oncologia no referido hospital e aos demais funcionários que foram prestativos para a realização desta pesquisa.

\section{REFERÊNCIAS}

1. Brasil. Conselho Federal da Farmácia. Resolução RDC no 288, de 21 de março de 1996. Diário Oficial da União. Brasília, DF, 1996 mai 17. [acesso em 2010 out 17]. Disponível em: http://www.cff. org.br/legis/pdf/288.pdf

2. Brasil. Ministério da Saúde. Portaria GM/MS n³. 535, de 02 de setembro de 1998. Estabelece critérios para cadastramento de centros de atendimento em oncologia. Diário Oficial da União. Brasília, DF, 1998 set 03; Seção 1, p75/77. [acesso em 2010 out 20]. Disponível em: www.saude.mg.gov.br/atos_normativos/ legislacao-sanitaria/estabelecimentos-de-saude/oncologia/portaria_3535.pdf

3. Brasil. Agência Nacional da Vigilância Sanitária. Resolução RDC n ${ }^{\circ} 220$, de 21 de setembro de 2004. Aprova o regulamento técnico de funcionamento dos serviços de terapia antineoplásicas. Diário Oficial da União. Brasília, DF, 2004 set 23. [acesso em 2010 out 14]. Disponível em: http://elegis.anvisa.gov.br/leisref/ public/showAct.php?id=12639

4. Sarzi PC Atenção Farmacêutica na Oncologia. [Internet]. 2012[acesso em 2013 fev 16]. Disponível em: http://hospitaldaprovidencia.org. br/noticias/207-atencao-farmcaceutica-na-oncolo gia.htm

5. Escobar GF, Gato MIR, Giorgenon AP Implementando processos em oncologia: diferenciais de qualidade e segurança. [Internet]. 2005. [acesso em 2010 out 25] Disponível em: http:// www.praticahospitalar.com.br/pratica\% 2041/ pags/artigos\%2041.html

6. Escobar GF Espaço do estudante. [Internet]. 2010. Sociedade Brasileira de Farmacêuticos em Oncologia - SOBRAFO. [acesso em 2010 out 10] Disponível em: http:// www.sobrafo.org.br

7. Andrade CC Farmacêutico em Oncologia: Interfaces Administrativas e Clinicas. Instituto do Câncer do Ceará. Fortaleza; 2009.
8. Nossa história. Sociedade Brasileira de Farmacêuticos em Oncologia - SOBRAFO. [Internet]. São Paulo; 2012. [acesso em 16 fev 2013]. Disponível em: www.sobrafo.org.br/?p=9

9. Santos L Credenciada Unidade de Alta Complexidade em Oncologia no Maranhão [Internet]. Imperatriz; 2011. [acesso em 2013 fev 16]. Disponível em: www.saude.ma.gov.br/ index.php?option $=$ com_conten $\& v i e w=\operatorname{article} \& i d=1730: \mathrm{cr}$ edenciada-unidade-de-alta-complexidade-em-ocologia-no-ma\&catid $=1$ :ultimas-noticias $\&$ Itemid $=800$

10. Guia digital de medicamentos genéricos. Agência Nacional de Vigilância Sanitária [Internet] 2006. [acesso em 2010 nov 10] Disponível em: http://www.pdamed.com.br/genericos/pdamed00030709.php

11. Rotea WJ Atuação do farmacêutico na oncologia: [Internet] 2007 [acesso em 2010 out 18] Disponível em: http://www.hcrp.frnrp. usp.br/ gxpfiles/arqs/pdf/farmacêutico\%20oncologiapdf

12. Martinez NVBV. Atenção farmacêutica. [Internet] [acesso em 2010 nov 11] Disponível em: http://www.farmaciauniversitaria. ufrj.br/ disciplinas/disciplina farmacia hospitalar/Atencao_Farmaceutica.pdf

13. Castro BP, Boechat L Desenvolvimento de ferramenta para o registro de reações adversas relacionadas a novos medicamentos oncológicos. [Internet] [acesso em 2010 nov 09] Disponível em: http://bvsms.saude.gov.br/bvs/publicacoes/inca/Bruno_desenvolvimento_de_ferramenta.pdf

14. Funes CM, Storrer JO Lidando com o paciente oncológico. [Internet] [acesso em 2010 nov 10] Disponível em: http://petfarmaciaufpr.files.wordpress.com/2010/11/lidando_com_o_paciente_oncologico.pdf 
15. Brasil Protocolos Clínicos e Diretrizes Terapêuticas do Ministério da Saúde [Internet] 2002 [acesso em 2010 nov 11] Disponível em: http://www.portal.saude.gov.br/portal/saude/.../visualizar_ texto.cfm?idtxt...1

16. Oliboni LS, Camargo AL Validação da prescrição oncológica: o papel do farmacêutico na prevenção de erros de medicação. Rev. HCPA \& Fac. Med. Univ. Fed. Rio Gd. do Sul; 29(2): 142- 152

17. Lara FC O papel da farmácia no âmbito hospitalar: desenvolvimento da assistência farmacêutica oncológica [Internet] 2009.29f. [Monografia] Escola de Saúde do Exército, Programa de Pós - Graduação em Aplicações Complementares às Ciências Militares, Rio de Janeiro, 2009.
18. Brasil Portaria MS/GM n1. 883, de 09 de setembro de 2008. Aprova o Formulário Terapêutico Nacional-FTN, da Relação Nacional de Medicamentos Essenciais - Rename 2008. Diário Oficial da União, p.47, 10 set. 2008. Seção 1.

19. Pepe VLE O Formulário Terapêutico Nacional, os Protocolos Clínicos e Diretrizes Terapêuticas: extensões da seleção de medicamentos. [Internet] [acesso em 2010 nov 15] Disponível em: http:// www.ensp.fiocruz.br/portal-ensp/judicializacao/pdfs/339/pdf 